\title{
Epidemiological profile and therapeutic approaches of bladder cancer patients referred to tertiary uro-oncology center
}

\author{
Nestor S. Penteado*, Ubirajara Ferreira, Wagner E. Matheus.
}

\section{Abstract}

Bladder cancer is the second most incident malignant tumor of the urinary tract and its histopathological stage is essencial to determine the appropriate therapeutic approach. Superficial tumors correspond to $70-80 \%$ of all cases and occur on caucasian men exposed to smoking or ocupacional chemical hazards.

The presente study's goal is to describe the epidemiological and histopathological profile, treatments perfomed, surgical and oncological outcomes in patients diagnosed with bladder cancer referred to a tertiary hospital with uro-oncology care center.

Key words: Bladder cancer, urinary tract, uro-oncology.

\section{Introduction}

Bladder cancer is the second most incident malignant tumor of the urinary tract and the ninth most incident type of cancer in the world. Considering its relevance in the oncological scenario, the present study aims to analyze epidemiological and histopathological profile, as well as therapeutic approaches, on patients diagnosed with bladder cancer treated in a tertiary referral hospital such as Hospital de Clínicas UNICAMP.

\section{Results and Discussion}

The method consists in retrospective study based on 271 chart review of patients that attended the UroOncology Department and were diagnosed with bladder cancer. When evaluating the collected data, information such as prevalence in age, gender and ethnic groups, as well as risk factors to the disease, were coincident with literature. Regarding histological description of tumors and oncological approach, results also matched.

Information that distanced from literature were postoperative complications in patients that underwent cystectomy and general mortality rates. It is possible to assume relation to professional learning curves, considering the institution is a teaching hospital, and the gravity of cases referred to a tertiary care hospital. Larger studies are needed to support these hypotheses.

\section{Conclusions}

The results found after gathering information demonstrate that patients referred to a tertiary hospital qualified in uro-oncology have similar demographical, epidemiological and therapeutic profiles as the literatures presently describes.

Further studies are recommended to better evaluate surgical complications and overall mortality in bladder cancer patients, specially in those with invasive and extensive tumors or with worse performance status at the diagnosis.

The present analysis also states its limitation due to retrospective bias.

\section{Acknowledgement}

The authors acknowledge the urology department and Hospital de Clínicas UNICAMP for the support regarding chart review and academical orientation.

Nardozza Júnior, A.; Zerati Filho, M.; Borges dos Reis, R. Urologia Fundamental. Traducao. 1. ed. São Paulo: Planmark Editora Ltda., 2010.

HOFF, Paulo Marcelo Gehm et al (Ed.). Tratado de Oncologia. São Paulo: Atheneu, 2012. 2860 p. 2 v

TORRE, Lindsey A. et al. Global cancer statistics, 2012. Ca: A Cancer Journal for Clinicians, [s.1.], v. 65, n. 2, p.87-108, 4 fev. 2015. American Cancer Society. http://dx.doi.org/10.3322/caac.21262. Disponível em: <https://www.ncbi.nlm.nih.gov/pubmed/25651787> 